\title{
Differential cerebral response to somatosensory stimulation of an acupuncture point vs. two non-acupuncture points measured with EEG and fMRI
}

\author{
Till Nierhaus ${ }^{1,2 *+}$, Daniel Pach ${ }^{3 *+}$, Wenjing Huang ${ }^{3,4+}$, Xiangyu Long ${ }^{2}$, Vitaly Napadow ${ }^{5,6}$, \\ Stephanie Roll ${ }^{3}$, Fanrong Liang ${ }^{4}$, Burkhard Pleger ${ }^{2}$, Arno Villringer ${ }^{1,2+}$ and Claudia M. Witt ${ }^{3,7 t}$
}

\footnotetext{
${ }^{1}$ Mind-Brain Institute at Berlin School of Mind and Brain, Charité - Universitätsmedizin Berlin and Humboldt-University, Berlin, Germany

${ }^{2}$ Department of Neurology, Max Planck Institute for Human Cognitive and Brain Sciences, Leipzig, Germany

${ }^{3}$ Institute for Social Medicine, Epidemiology, and Health Economics, Charité - Universitätsmedizin Berlin, Berlin, Germany

${ }^{4}$ Acupuncture and Tuina School, Chengdu University of Traditional Chinese Medicine, Chengdu, China

${ }^{5}$ Department of Radiology, Athinoula A. Martinos Center for Biomedical Imaging, Massachusetts General Hospital, Charlestown, MA, USA

${ }^{6}$ Department of Radiology, Logan University, Chesterfield, MO, USA

7 Institute for Complementary and Integrative Medicine, University Hospital Zurich, Zurich, Switzerland
}

\author{
Edited by: \\ Leonhard Schilbach, University \\ Hospital Cologne, Germany \\ Reviewed by: \\ Matthew R. Longo, Birkbeck, \\ University of London, UK \\ Philipp Georg Sämann, Max Planck \\ Institute of Psychiatry, Germany

\section{*Correspondence:} \\ Till Nierhaus, Mind-Brain Institute \\ and Berlin School of Mind and Brain \\ Humboldt-University, Luisenstraße \\ 56, 10117 Berlin, Germany \\ e-mail: till.nierhaus@charite.de; \\ Daniel Pach, Institute for Social \\ Medicine, Epidemiology, and Health \\ Economics, Charité - \\ Universitätsmedizin Berlin, 10098 \\ Berlin, Germany \\ e-mail:daniel.pach@charite.de \\ ${ }^{t}$ These authors have contributed \\ equally to this work.
}

Acupuncture can be regarded as a complex somatosensory stimulation. Here, we evaluate whether the point locations chosen for a somatosensory stimulation with acupuncture needles differently change the brain activity in healthy volunteers. We used $E E G$, event-related $f M R I$, and resting-state functional connectivity $f M R I$ to assess neural responses to standardized needle stimulation of the acupuncture point ST36 (lower leg) and two control point locations (CP1 same dermatome, CP2 different dermatome). Cerebral responses were expected to differ for stimulation in two different dermatomes (CP2 different from ST36 and CP1), or stimulation at the acupuncture point vs. the control points. For EEG, mu rhythm power increased for ST36 compared to CP1 or CP2, but not when comparing the two control points. The $\mathrm{fMRI}$ analysis found more pronounced insula and S2 (secondary somatosensory cortex) activation, as well as precuneus deactivation during ST36 stimulation. The S2 seed-based functional connectivity analysis revealed increased connectivity to right precuneus for both comparisons, ST36 vs. CP1 and ST36 vs. CP2, however in different regions. Our results suggest that stimulation at acupuncture points may modulate somatosensory and saliency processing regions more readily than stimulation at non-acupuncture point locations. Also, our findings suggest potential modulation of pain perception due to acupuncture stimulation.

Keywords: somatosensory stimulation, functional magnetic resonance imaging (fMRI), electroencephalography (EEG), acupuncture, background rhythm, functional connectivity

\section{INTRODUCTION}

In a recent patient level data meta-analyses for chronic pain that included 29 randomized controlled trials, acupuncture was shown to be statistically significant superior to sham acupuncture (Vickers et al., 2012). Nevertheless, as the difference in effect size between real and sham acupuncture was small (standard mean difference of 0.15-0.23), the acupuncture point-specific effect is still controversial. One reason might be that various forms of sham acupuncture have been used in previous clinical trials. Often a penetrating sham acupuncture has been applied where either the control point location is different (Ma et al., 2012) (not a specific acupuncture point), the method of the stimulation is changed (Kleinhenz et al., 1999) (e.g., only superficial needling, no manually rotating and lift-thrusting), or both (Diener et al., 2006). Sanchez-Araujo (1998) showed in his review that studies where the control points were chosen to be near the real acupuncture points failed more frequently to show statistically significant differences between real acupuncture and sham acupuncture compared to using control acupuncture points located in different dermatomes (Sanchez-Araujo, 1998). In the past decade there has been growing interest in determining the neurophysiologic correlates of acupuncture by means of functional neuroimaging methods such as fMRI or EEG (Hui et al., 2000; Dhond et al., 2007; Hori et al., 2010; Huang et al., 2012). From a neurophysiological viewpoint, acupuncture is regarded as a complex somatosensory stimulation (Bäcker et al., 2004) and therefore is also interesting for experiments using conventional somatosensory stimulation protocols.

The purpose of this study was to evaluate whether the point locations chosen for a complex somatosensory stimulation with acupuncture needles differentially impact brain activity in healthy volunteers. We used electroencephalogram (EEG) and functional magnetic resonance imaging (fMRI) to compare standardized needle stimulation on three different point locations on the right leg: one acupuncture point (Stomach 36, ST36) and two control points that are widely accepted to not co-localize with points in 
any acupuncture system. One of these control points was chosen to be near the real acupuncture point in the same dermatome L5 (CP1), while the other was chosen to be in a different dermatome L2 (CP2). Imaging results are expected to differ either when comparing the two different dermatomes (CP2 different from ST36 and CP1), or when comparing the acupuncture point with the two non-acupuncture control points.

Using fMRI, the blood oxygenation level dependent (BOLD) signal can localize brain regions modulated by sensory stimulation (Bandettini et al., 1992; Frahm et al., 1992; Kwong et al., 1992; Ogawa et al., 1992; Kurth et al., 2000; Ruben et al., 2001). Several studies have demonstrated acupuncture-related brain activity changes in somatosensory and pain-related areas such as primary somatosensory cortex (S1), secondary somatosensory cortex (S2), and thalamus (Napadow et al., 2009; Huang et al., 2012). Also, functional connectivity MRI (fcMRI) revealed acupuncture-related changes in network connectivity between areas related to sensorimotor and pain processing (Dhond et al., 2008). Therefore, stimulation of the acupuncture point might specifically activate brain regions associated with pain and modulate their connectivity. Using EEG, stimulation effects can be shown by investigation of background rhythmic activity, in the somatosensory system the "mu rhythm," respectively (Gastaut, 1952; Kuhlman, 1978). While the precise function of the mu rhythm is not clear, it is frequently referred to as an "idle rhythm" reflecting a resting-state of the somatosensory system (Ritter et al., 2009). Recent studies assume that EEG background activity is produced by inhibitory inter-neuronal activity and might reflect inhibitory top-down control (Klimesch et al., 2007; Jensen and Mazaheri, 2010). With respect to the clinical effectiveness shown for pain conditions, the stimulation of the acupuncture point might inhibit somatosensory areas and therefore increase mu-rhythmic activity.

We designed a blinded study including two series of experiments (one with EEG, one with fMRI) focusing on brain activity changes associated with complex somatosensory stimulation at one acupuncture point and two control points either close or distant to the acupuncture point. Our study was not aimed at evaluating clinical effects of acupuncture. We believe that our data provide new insights regarding cerebral processing of complex somatosensory stimulations and clarify the role of stimulus location for brain responses to complex somatosensory stimulation.

\section{MATERIALS AND METHODS SUBJECTS}

Twenty-three healthy subjects (11 female, 12 male, mean age 26 years, range 19-31 years) participated in the EEG experiment, and 22 healthy subjects (11 female, 11 male, mean age 26 years, range 21-32 years) participated in the fMRI experiment. Participants had no medical knowledge about acupuncture and all except one had never been treated with acupuncture before the study. Participants were informed about the needle stimulation in both experiments as follows: “... one acupuncture needle will be inserted into the muscle at three different points of the upper and lower leg..." Among all the subjects, eight participated in both EEG and fMRI experiments.
All participants were right-handed (laterally score: $88.2 \pm 13.4$ [S.D.] over a range of -100 [fully left-handed] to 100 [fully right-handed]) according to the Edinburgh inventory (Oldfield, 1971) and gave written informed consent to participate in the experiment according to the declaration of Helsinki. The study was approved by the ethics committee of the University of Leipzig. Prior to participation all subjects underwent a comprehensive neurological examination and confirmed they were not taking any acute or chronic medication. In the EEG experiment three subjects were excluded from acupuncture because of vegetative side effects ( 1 sweating/male, 2 dizziness/female); in the fMRI experiment one was excluded (sweating/female).

\section{DETAILED DESCRIPTION OF POINT LOCATIONS}

The points chosen for the intervention were developed after literature screening and a consensus process between the authors and experts of the Chengdu University of TCM, Prof Liang and Prof Li (Figure 1A).

1. Acupuncture point ST36 (Zusanli 足三里):

To locate ST36, which is placed on the anterior lower leg, the acupuncture points ST35 and ST41 are used as anatomical landmarks. ST36 is located on the line connecting ST35 with ST41, 3 B-cun inferior to ST35 (ST35 is located on the anterior aspect of the knee, in the depression lateral to the patellar ligament. ST41 is located on the anterior aspect of the ankle, in the depression at the center of the front surface of the ankle joint, between the tendons of extensor hallucis longus and extensor digitorum longus) (Who Regional Office for the Western Pacific, 2008). According to Chinese medicine theory, for healthy subjects, ST36 is a commonly used acupuncture point to strengthen Qi and blood as a health preservation application. ST36 is located on the stomach meridian within the stomach meridian area. The skin area of ST36 belongs to L5 dermatome (Yan, 2006).

2. Control point 1 (located in the same dermatome and not in the same meridian skin area):

The point is located lateral to the ST36 horizontally, at the middle line between Bladder meridian and Gallbladder meridian. Control point 1 is selected according to the principle of selecting non-acupuncture points from the middle line between two meridians which is commonly used in Chinese studies (Yang et al., 2009). Control point 1 is located in L5 dermatome and between Gallbladder and Bladder meridian skin area.

3. Control point 2 (located in another dermatome and not in the same meridian skin area):

The point is located 2 B-cun dorsally of GB31 (avoidance of bladder meridian: GB31 is located on the lateral aspect of the thigh, in the depression posterior to the iliotibial band where the tip of the middle finger rests when standing up with the arms hanging alongside the thigh). Control point 2 is already a validated non-acupuncture point used in other acupuncture studies (Brinkhaus et al., 2003; Melchart et al., 2005; Linde et al., 2006) and it is located in L2 dermatome and Gallbladder meridian skin area. 


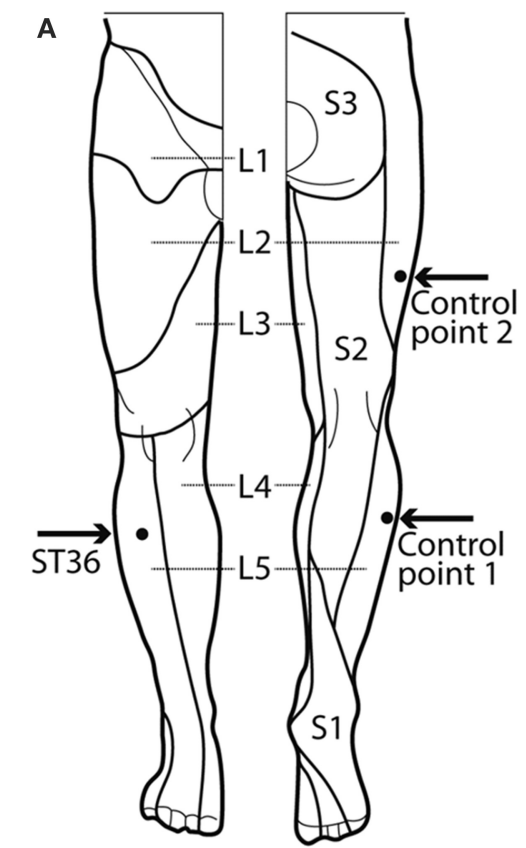

B
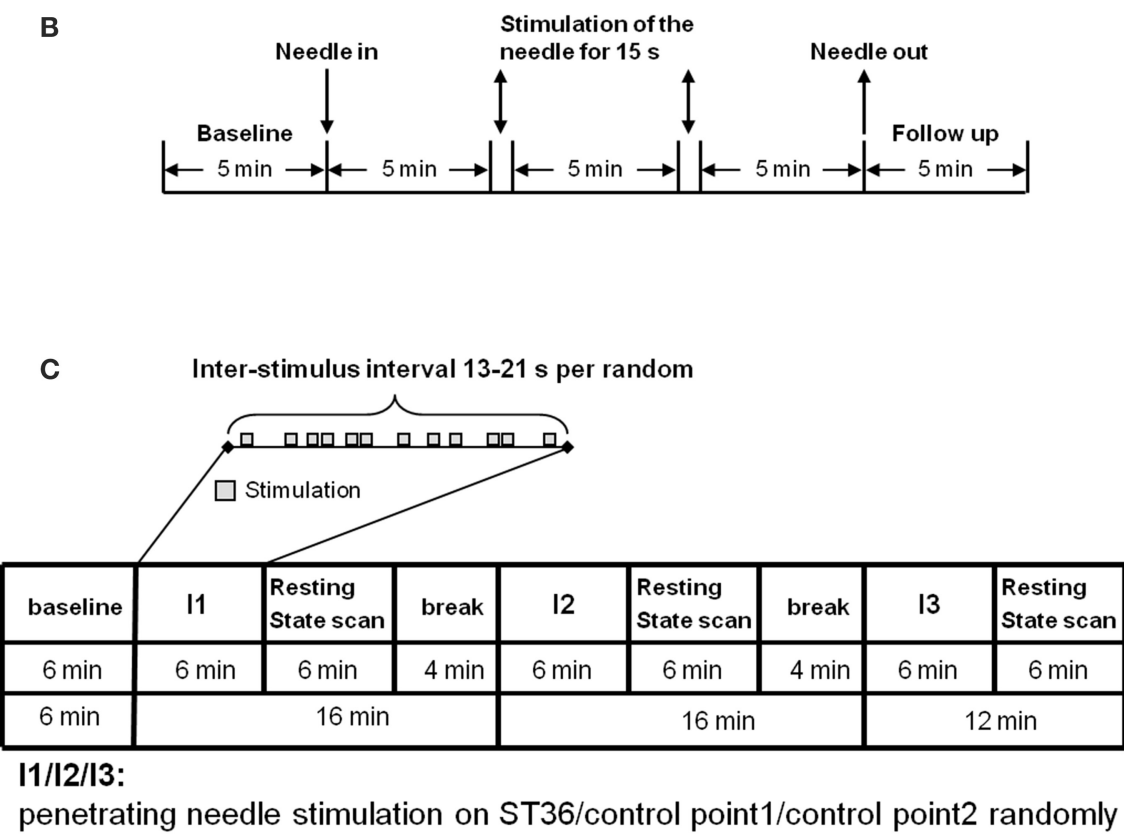

FIGURE 1 | Experimental design. (A) Location of ST36 and Control points on the right leg (view from the front and from the back, figure adapted Drake et al., 2009). (B) Experiment design of EEG measurement. On each of the 3 randomized measurement days EEG was recorded for every subject in a sitting position, with open eyes over a 25.5 -min period. After the first 5-min baseline, an acupuncture needle was inserted and was stimulated manually at a time point 5 and $10 \mathrm{~min}$ after insertion. After a further $5 \mathrm{~min}$ the needle was removed and the EEG was recorded for a further 5 min without the needle. After the measurement, individual needle sensation was measured with a questionnaire. (C) Experiment design of $\mathrm{fMRI}$ measurement. In the $\mathrm{fMRI}$ scanner each subject was told to lie relaxed, in a supine position, with open eyes and to concentrate on the sensation caused by the needle stimulation. After the first resting-state was scanned for $6 \mathrm{~min}$, an acupuncture needle was inserted into one point according to the randomized intervention order. The needle was then immediately stimulated manually according to the event-related design. After 6 min intermittent manipulation, the needle was withdrawn and a resting-state scan was continued for $6 \mathrm{~min}$, and then followed by a 4-min break for the subject without scanning. The same scan procedure was then applied for the other two interventions on the other two points. But for the third intervention, there was no 4-min break. After each post-intervention resting-state scan individual needle sensation was measured with questionnaires.

\section{EEG}

\section{Experimental Procedure}

All subjects received the needle stimulation of the three different points (Figure 1) - the acupuncture point ST36 in dermatome L5, the control point in the same dermatome (CP1 in L5), the control point in a different dermatome (CP2 in L2)-on 3 separate days in consecutive order. The order was randomized. Measurements were taken within 2 weeks and with at least $24 \mathrm{~h}$ interval between each measurement. Subjects were told to sit down in a chair in the EEG room and relax with eyes open while concentrating on the point of needling.

The penetrating needle stimulation was performed by a Chinese acupuncture physician with sterile, single use, individually wrapped acupuncture needles $(0.30 \times 30 \mathrm{~mm}$; asia-med standard, asia-med GmbH \& Co. KG, Germany). The needle was vertically inserted $1-2 \mathrm{~cm}$ deep into the skin depending on the size of the respective muscle on the right leg. After 5 min the needle was stimulated (manually rotating 60-90/rpm and lift-thrusting $0.3-0.5 \mathrm{~cm}$ for $15 \mathrm{~s}$ ). The $15 \mathrm{~s}$ stimulation was repeated after $5 \mathrm{~min}$ without stimulation. Penetrating needle stimulation was identical for each of the three point locations (Figure 1).

\section{Data acquisition}

A 32-channel EEG was recorded in a noise protected and electrically shielded room using BrainAmp (Brain Product, Germany) with a sampling rate of $1000 \mathrm{~Hz}$. An electrode cap (Electro-Cap International, Eaton, $\mathrm{OH}$ ) based on the international 10-20 system was placed on the scalp. The electrode $\mathrm{FCz}$ was used as reference and the ground electrode was located at the sternum. Electrode impedances were less than $2 \mathrm{kOhm}$. Including the 5min baseline, the intervention, and the follow-up measurements the EEG was recorded for $25.5 \mathrm{~min}$.

After each measurement the subjects were asked to fill in the MGH Acupuncture Sensation Scale (MASS questionnaire Kong et al., 2007) to measure the subjective needle sensation.

\section{Data preprocessing}

For data analysis custom-built scripts in the software package Matlab (Matlab, MathWorks, Inc.) were used. Since somatosensory alpha activity (Rolandic activity) can be covered by strong occipital alpha activity, an independent component analysis (ICA) was performed to allow for a preselection of "central" ICA components. For each subject the three sessions were merged to perform one ICA calculation (FastICA algorithm in Matlab). Rolandic rhythmic activity is characterized by a central 
localization and a peak in the frequency spectrum in the alpha $(8-15 \mathrm{~Hz})$ and beta $(16-30 \mathrm{~Hz})$ range. Thus, ICA components were investigated for each subject and selected only if both a central topography and two peaks in the frequency spectrum were identifiable. Using this procedure, $2-10$ (mean $5 \pm 2$ S.D.) central components were selected per subject. The selected central components were back projected and the derived dataset (now cleared from occipital alpha) was digitally filtered using a standard 3rd order band-pass Butterworth filter (low cut-off $1 \mathrm{~Hz}$, high cut-off $45 \mathrm{~Hz}$ ) and segmented into 5 epochs each lasting $4 \mathrm{~min}$, based on the markers representing the interventions. Since we performed needle stimulation on the leg further data analysis was focused on electrode $\mathrm{Cz}$ which is located over the leg representation of $\mathrm{S} 1$. Frequency analysis was performed using fast Fourier transformation. The power spectral density was computed for each 4-min segment: (1) for baseline, (2) after the "needle-insertion," (3) after the first stimulation, (4) after the second stimulation, and (5) for follow up. For the statistical analysis of the mu activity, power spectral density was averaged for the frequencies from $10 \mathrm{~Hz}$ to $15 \mathrm{~Hz}$.

\section{Statistical analysis}

For $\mathrm{Cz}$ electrode and each condition, the mu power change and percentage change compared to baseline was analyzed using generalized linear models for our within subject design with global $F$-tests and paired $t$-tests for pair-wise comparisons between stimulation points. For our primary outcome parameter, the mean of both post-stimulation periods, a Bonferroni correction was applied to the pair-wise comparison between the three stimulation points $\left(p \mathrm{R}_{\text {corr }}=0.05 / 3\right)$.

To evaluate the correlation between alpha percentage change from baseline and needle sensation (MASS Index), Spearman correlation coefficients based on the ranks of the variables were used for $\mathrm{Cz}$ electrode, each condition, and each needle stimulation.

The needle sensation expressed by the MASS Index was compared descriptively for the three needle stimulations at different points by presenting means and $95 \%$ confidence intervals. To test a global stimulation point effect (within-subject effect) on the MASS Index, generalized linear models (GLM) were fitted using a multivariate approach (Wilks' lambda) because sphericity was often not met. To test pair-wise differences between the three points, paired $t$-tests were used.

\section{fMRI}

\section{Experimental Procedure}

As shown in Figure 1C, each participant was scanned seven times (each scan $6 \mathrm{~min}$ ): One resting-state scan in the very beginning (i.e., baseline, RS_B), then three scans with needle stimulation of one point in an event-related design, each followed by another resting-state scan (i.e., RS_ST36, RS_CP1 and RS_CP2). The three event-related scans were in randomized order over subjects. During scanning, subjects were told to remain in the supine position with eyes open while concentrating on the sensation caused by the needle stimulation. During the resting-state, participants were simply asked to keep calm and stay still with eyes open.

The penetrating needle stimulation was performed by an acupuncture physician with sterile, single use, individually wrapped needles $(0.20 \times 30 \mathrm{~mm}$; titanium, DongBang,
Acupuncture, Inc., Boryeong, Korea). The needle was first inserted $1-2 \mathrm{~cm}$ deep into the skin depending on the size of the muscle vertically on the right leg. The needle was manually manipulated according to the event-related design starting immediately after insertion. Auditory cues signaled the timing of the stimulation events to the acupuncturist via headphones. Each event consisted of a 3-s needle stimulation rotating $60-90 / \mathrm{rpm}$ and lift-thrusting $0.3-0.5 \mathrm{~cm}$. The length of the inter-stimulus interval was randomized from 13 to $21 \mathrm{~s}$ (Figure 1B). After the event-related scan the needle was removed. Identical penetrating needle stimulation was performed on the three different point locations (Figure 1).

\section{Data acquisition}

fMRI Data was acquired using a 3T Siemens Verio MRI System (Siemens Medical, Erlangen, Germany) equipped for echo planer imaging with a 12-channel head coil. fMRI images were acquired using an EPI sequence (30 axial slices, in-plane resolution is $3 \times 3 \times 5 \mathrm{~mm}$, slice thickness $=4 \mathrm{~mm}$, flip angle $=90^{\circ}$, gap $=$ $5 \mathrm{~mm}$, repetition time $=2000 \mathrm{~ms}$, echo time $=30 \mathrm{~ms}$ ). A structural image was also acquired for each participant, using a T1-weighted MPRAGE sequence (repetition time $=12 \mathrm{~ms}$, echo time $=5.65 \mathrm{~ms}$, and flip angle $=19^{\circ}$, with elliptical sampling of $\mathrm{k}$ space, giving a voxel size of $1 \times 1 \times 1 \mathrm{~mm})$. Subjects' heads were immobilized by cushioned supports, and they wore earplugs to attenuate MRI gradient noise throughout the experiment.

Within the break following resting-state scans, subjects were asked to rate the items of the MGH Acupuncture Sensation Scale (MASS questionnaire Kong et al., 2007) to measure the subjective needle sensation.

\section{Data pre-processing}

FMRI data pre-processing included slice time correction, head motion correction, spatial normalization to MNI152 space and spatial smoothing with a $6 \mathrm{~mm}$ FWHM as implemented in the SPM 8 software package (www.fil.ion.ucl.ac.uk/spm/). Individual structure T1 images were also normalized to MNI152 space and then segmented into gray matter, white matter and cerebral spinal fluid (CSF). A threshold of 0.99 was used to cut off each segmented image. For each participant, $3 \mathrm{~mm}$ erosion was implemented on the white matter image and $1 \mathrm{~mm}$ erosion on the CSF image, and these two images were then combined into one anatomical mask. We applied principal component analysis (PCA) within this CSF/white matter mask to disentangle the variance related to each $\mathrm{fMRI}$ dataset (3 task scans and 4 restingstate scans) using the CompCor analysis (Behzadi et al., 2007) by DPABI toolbox (toolbox for Data Processing \& Analysis of Brain Imaging, http://rfmri.org/dpabi). The first five principal components together with the six head motion parameters were later applied to each individual's first level GLM as nuisance variables to regress out associated variance. A union gray matter mask (Supplemental Figure 1) was created by merging all normalized individual gray matter images. The following analyses were conducted within this average gray matter mask.

\section{GLM analysis}

For each subject the first-level GLM contained the three different needle stimulation conditions (i.e., ST36, CP1, CP2; 6 min 
stimulation for each point). For each condition, one stimulation regressor, together with the first five principal components from the CompCor analysis and six head motion parameters as nuisance regressors were included in the GLM. For the stimulation regressor, each stimulation onset was modeled with the boxcar function covering the following $3 \mathrm{~s}$ stimulation duration. These box-car functions were convolved with the standard hemodynamic response function (HRF) as implemented in SPM 8. The long inter-stimulus intervals of 13-21 s were not explicitly modeled with the first level GLMs and hence represented an implicit baseline measure. For each of the three stimulation points we computed the individual $ß$-map.

On the second level (group) analyses, the main effect of each of the three stimulation points (i.e., ST36, CP1, CP2) was visualized by applying these individual $B$-maps (together with age and gender as covariates) to one-sample $t$-tests to compare them against the null hypothesis. The results were corrected to the alpha-level <0.05 using AlphaSim in AFNI (Cox, 1996) (i.e., 39429 voxels within the gray matter mask, voxel-wise $p<0.0001$, resulting cluster size $>108 \mathrm{~mm}^{3}$ ). We performed a within subjects ANOVA (factorial design within SPM8) including the individual B-maps of all three conditions (ST36, CP1, CP2) as well as age and gender as covariates to generate the inter-points comparisons (i.e., ST36-CP1, ST36-CP2 and CP1-CP2) and conjunction maps within one statistical model. Conjunction of "ST36-CP1 and ST36-CP2" was calculated to compare the activation between acupuncture and control points, conjunction of "ST36-CP2 and CP1-CP2" was calculated to compare activations of the different dermatomes (L5 vs. L2). Using AlphaSim in AFNI, the results for the interpoint-comparisons as well as for the conjunctionmaps were corrected to the alpha-level $<0.05$ (i.e., 39429 voxels within the gray matter mask, voxel-wise $p<0.01$, resulting cluster size $>783 \mathrm{~mm}^{3}$ ). We used " 3 dclust" in AFNI to detect clusters from the corrected statistical maps. All clusters that were reported in the tables are spatially separated and bigger than the volume criterion from the AlphaSim simulation analysis.

\section{Functional connectivity analyses}

For each of the four resting-state scans (RS_B, RS_ST36, RS_CP1, and RS_CP2) the first 10 volumes were discarded to account for the saturation of the BOLD response. Temporal band-pass filtering $(0.01-0.08 \mathrm{~Hz})$ and removal of linear trend was performed by the REST toolbox (www.restfmri.net). Seed-based voxel-wise functional connectivity analysis was performed for each restingstate scan using region of interest (ROI) spheres with $6 \mathrm{~mm}$ radius as seeds. As a proof of concept, one seed was placed on posterior cingulate cortex (PCC, Talairach space, $x=-2, y=-36, z=37$ from a previous study Fox et al., 2005) that, as hypothesized, revealed the default mode network (Supplemental Figure 1). The other seed was derived from a group-level one-sample $t$-test across all three stimulation points together, with the maximum found at the parietal operculum as the anatomical site of S2 (Talairach space, $x=-54, y=-21, z=21$ ).

Then, the average time course within the ROI was extracted as the seed signal, and a voxel-wise temporal correlation analysis was performed across all voxels within the averaged gray matter mask for each individual resting-state scan. The correlation maps were transferred to Fisher's $\mathrm{z}$ maps for further statistical analysis (Greicius et al., 2007). First, a one-sample $t$-test against null hypothesis was performed on the spatial correlation maps of each resting-state scan (together with age and gender as covariates) for the PCC-seed and the S2-seed, respectively. The results were corrected to the alpha-level $<0.05$ using AlphaSim in AFNI (i.e., 39429 voxels within the gray matter mask, voxelwise $p<0.0001$, resulting cluster size $>108 \mathrm{~mm}^{3}$ ). Again, we performed a within subjects ANOVA including the individual spatial correlation maps of all four resting-state scans as well as age and gender as covariates. Within this model, we generated the comparisons to baseline (RS_ST36-RS_B, RS_CP1-RS_B, and RS_CP2-RS_B), the inter-points comparisons (RS_ST36RS_CP1, RS_ST36-RS_CP2, and RS_CP1-RS_CP2), and the conjunction maps. Conjunction of "RS_ST36-RS_CP1 and RS_ST36-RS_CP2" was calculated to compare functional connectivity between acupuncture and control points, conjunction of “RS_ST36-RS_CP2 and RS_CP1-RS_CP2" was calculated to compare connectivity between the different dermatomes (L5 vs. L2). Using AlphaSim in AFNI, the results for the different comparisons as well as for the conjunction-maps were corrected to the alpha-level $<0.05$ (i.e., 39429 voxels within the gray matter mask, voxel-wise $p<0.01$, resulting cluster size $>783 \mathrm{~mm}^{3}$ ). As described above, we used " 3 dclust" in AFNI to detect clusters from the corrected statistical maps.

\section{Needle sensation analyses}

The needle sensation expressed by the MASS Index was compared descriptively for the three needle stimulations at different points by presenting means and $95 \%$ confidence intervals. To test a global stimulation point effect (within-subject effect) on MASS Index, generalized linear models (GLM) were fitted using a multivariate approach (Wilks' lambda) because sphericity was often not met. To test pair-wise differences between the three points, paired $t$-tests were used.

For each region that was detected in the conjunction analyses (Figures 3C,D), Pearson correlation coefficients were calculated across participants between the mean beta value across voxels within the respective region and the MASS index of each stimulation point.

\section{RESULTS}

EEG

\section{Mu rhythm}

The mu rhythm is one of the important human brain background rhythms and is associated with the primary somatosensory area, thus having a central topography (Salmelin and Hari, 1994). In healthy volunteers we stimulated the three different points mentioned above in the same manner and compared their respective influences on mu rhythm. Data is shown for electrode $\mathrm{Cz}$ which is closest to the lower limb representation in S1. Mu rhythm power was significantly enhanced after stimulation of ST36 compared to the stimulation of the two control points (mean of stimulation phase 1 and phase 2 vs. baseline: ST36 vs. $\mathrm{CP} 121.02 \mu \mathrm{V}^{2}$ 95\%CI [4.78;37.27], $p=0.012$, ST36 vs. CP2 $25.38[9.12 ; 41.65], p=0.003$, significance level Bonferroni corrected $0.05 / 3$ ). Comparison of mu rhythm for the two control 
points found no significant differences (CP2 vs. CP1 -4.36 $[-20.53 ; 11.81], p=0.598$, Figure 2).

\section{Needle sensation}

As our results for the mu rhythm may have been influenced by differences in needle sensation, the evoked sensation was measured using the MGH Acupuncture Sensation Scale (MASS Kong et al., 2007).

The MASS Index was used as a measure of needle sensation for ST36, CP1, and CP2 (3.15 [2.00;4.30], 3.37 [2.47;4.28], and 1.81 $[1.13 ; 2.50]$, respectively). Comparisons of ST36 vs. CP2 and CP1 vs. CP2 were statistically different (pairwise $t$-test, $p=0.034$ and $p<0.001$, respectively). However, a significant difference was not found between ST36 and CP1 $(p=0.674)$.

No correlations were found when exploring the relationship between the MASS index and the percentage change of mu rhythm power (all $r$-values between -0.16 and 0.40 with $p>0.080)$.

\section{FMRI}

\section{Stimulation scans}

The results of the intra-point analysis summarizing the BOLD response to needle stimulation of ST36 and the two control points are shown in Figure 3A and Table 1. For all three point stimulations we found significant activation in bilateral insula/S2 and left inferior semi-lunar lobule and deactivation in bilateral precuneus, right middle temporal gyrus, left superior frontal gyrus, right precentral gyrus, left medial frontal gyrus, right paracentral lobule, and bilateral parahippocampal gyrus.

We compared BOLD responses of the different points (ST36 vs. $\mathrm{CP} 1, \mathrm{ST} 36$ vs. $\mathrm{CP} 2$, and $\mathrm{CP} 1$ vs. $\mathrm{CP} 2$, shown in Figure 3B and Table 2) and with a conjunction analysis we evaluated shared areas for the comparison of acupuncture point vs. control points (conjunction of ST36-CP1 and ST36-CP2) and the comparison of two different dermatomes L5 and L2 (conjunction of ST36-CP2 and CP1-CP2).

For the comparison between acupuncture point and control points (ST36-CP1 and ST36-CP2), the conjunction analysis (Figure 3C, Table 2) revealed that right insula and right S2 presented higher activation during stimulation of ST36. The right precuneus/posterior cingulate cortex (PCC) presented pronounced deactivation during stimulation of the acupuncture point.

For the comparison between the dermatomes (ST36-CP2 and CP1-CP2), a common positive contrast was shown for right middle temporal gyrus (MTG) due to deactivation during stimulation of CP2 compared to activation when stimulating the other two points (Figure 3D, Table 2). Left superior temporal gyrus (STG) presented pronounced deactivation when stimulating ST36 or $\mathrm{CP} 1$ compared to stimulation of CP2.

\section{Resting-state scans}

At the first stage, the default mode network and somatosensory network were detected via the seed-based correlation analysis within the resting-state scans. By visual inspection, PCC, $\mathrm{mPFC}$ and bilateral angular gyrus (prominent marker of the default mode network) were all found in the PCC-seed-based correlation analysis (Supplemental Figure 1). For the S2-seedbased correlation analysis, we found bilateral S2, supplementary motor area (SMA), and bilateral S1/M1 as prominent areas corresponding to the somatosensory network (Supplemental Figure 1, Table 3). The results of the S2-seed-based correlation analysis were further compared between the different resting-state scans.

The different brain areas that showed changes in functional connectivity after stimulation of the three different points

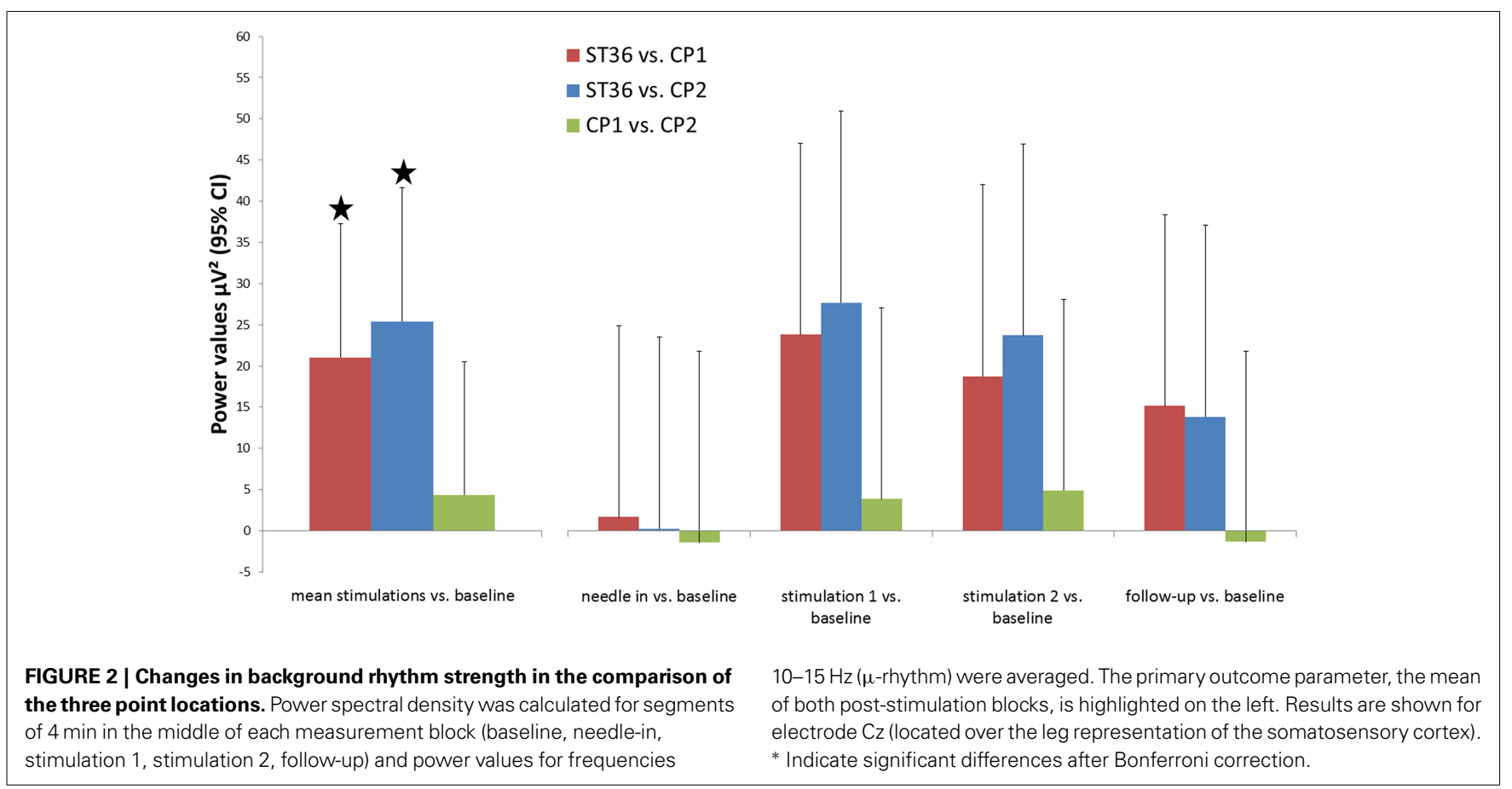




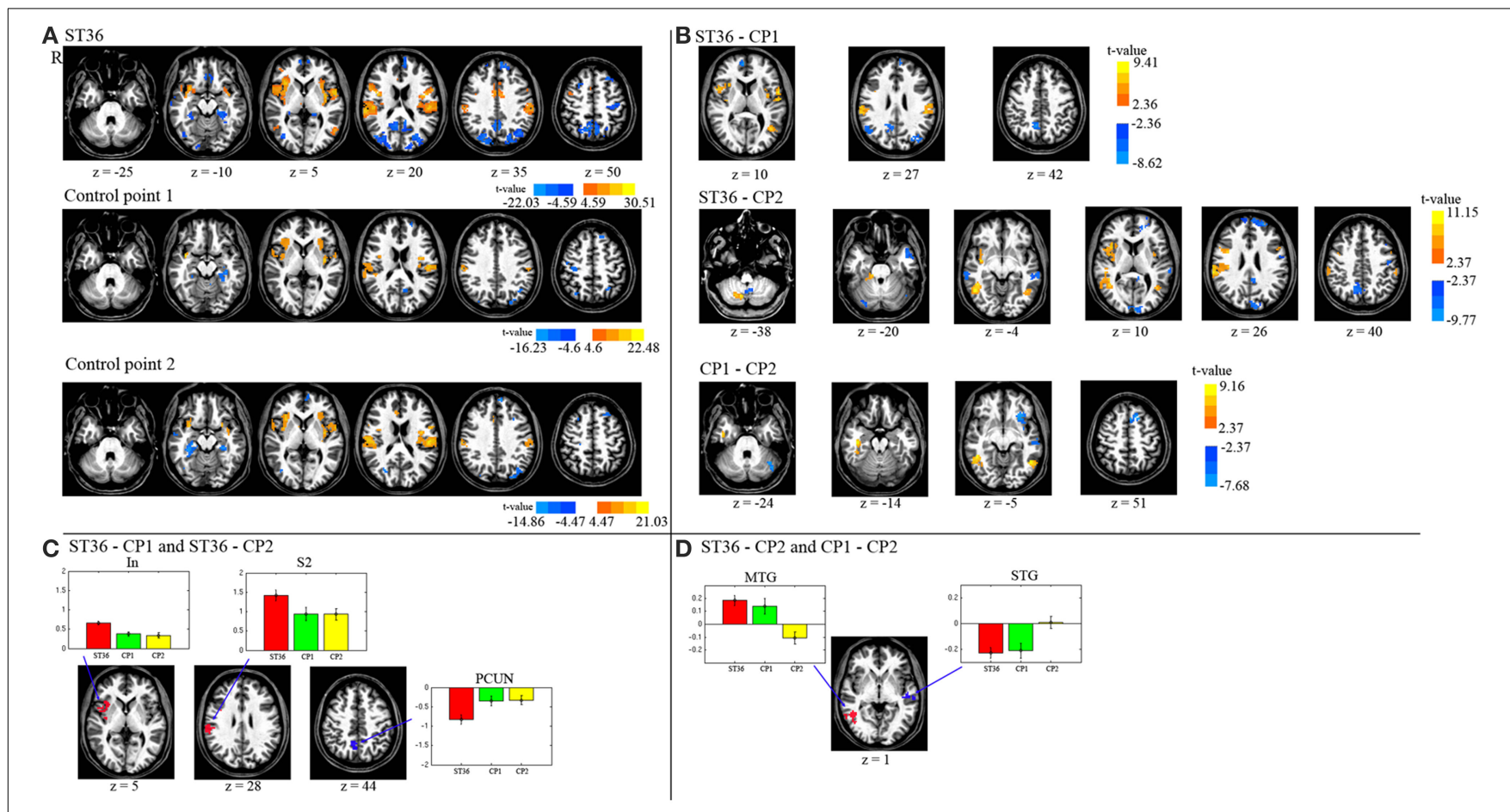

FIGURE 3 | (A) Displayed are the activation and deactivation for the different point stimulations (group-level t-maps, $P<0.05$, corrected). (B) The de/activation contrasts between the three different point locations are presented $(P<0.05$, corrected). (C) The conjunction map of the 1st and 2nd row of part (B) (acupuncture vs. control points). (D) The conjunction map of the 2nd and 3rd row of part (B) (dermatome L5 vs. L2). The barplots show the beta values across participants (average and standard error) within the respective region. $\mathrm{R}$ means right hemisphere. Talairach $\mathrm{z}$ coordinates are displayed. positive values: red, negative: blue. compared to the baseline resting-state session are depicted in Table 4 and Figure 4A.

ST36 as compared to CP1 revealed a significantly enhanced S2connectivity to right precuneus, right MTG, and right parahippocampal gyrus (Figure 4B, Table 4).

ST36 as compared to CP2 showed a significantly enhanced S2-connectivity to right precuneus/cuneus and right culmen, whereas the left medial frontal gyrus, left inferior frontal gyrus, and left superior temporal gyrus showed a significantly reduced connectivity to S2 (Figure 4B, Table 4).

Comparing the two control points $(\mathrm{CP} 1-\mathrm{CP} 2)$ revealed a significantly reduced S2-connectivity to right parahippocampal gyrus, left precuneus, and left superior temporal gyrus (Figure 4B, Table 4).

The conjunction analyses of the seed-based resting state connectivity for the comparison of acupuncture point vs. control points (conjunction of RS_ST36-RS_CP1 and RS_ST36-RS_CP2), as well as the comparison of two different dermatomes L5 and L2 (conjunction of RS_ST36-RS_CP2 and RS_CP1-RS_CP2) revealed no commonly change in connectivity.

\section{Needle sensation}

Similar to the EEG experiment the needle sensation was also assessed with the MASS (Kong et al., 2007). The MASS Index for ST36, CP1 and CP2 were 4.71 [3.53;5.89], 3.59 [2.51;4.68], and 3.32 [2.34;4.29], respectively. Differences between ST36 vs. CP1 and ST36 vs. CP2 were statistically significant (pairwise $t$-test, $p=0.009$ and $p=0.005$, respectively). There was no significant difference between CP1 and CP2 $(p=0.587)$.

No correlation was found when exploring the relationship between the MASS index and the mean beta values within the ROIs detected in the conjunction analysis (all r-values between -0.48 and 0.51 with $p>0.05$, corrected for multiple comparison).

\section{DISCUSSION}

We compared the stimulation of the acupuncture point ST36 with two control points that were non-acupuncture points: one near the real acupuncture point in the same dermatome (CP1 in L5) and one in a different dermatome (CP2 in L2). We expected the EEG and fMRI imaging results to be different either when comparing the points in the two different dermatomes (CP2 different from ST36 \& CP1), or when comparing the acupuncture point with the two non-acupuncture control points. Comparisons between points in the two different dermatomes (ST36 vs. CP2 and $\mathrm{CP} 1$ vs. $\mathrm{CP} 2$ ) showed more pronounced activation at right middle temporal gyrus and deactivation at left superior temporal gyrus when stimulating dermatome L5 (ST36 or CP1). When comparing the acupuncture point with the control points (ST36 vs. CP1 and ST36 vs. CP2) we found (i) pronounced BOLD activation in right insula and right $\mathrm{S} 2$, pronounced deactivation in precuneus/PCC, and (ii) a pronounced increase of mu rhythm power in the EEG data following stimulation of ST36. Moreover, increased connectivity of left S2 to the right precuneus was 
Table 1 | Foci with significant BOLD response from the three points $(P<0.05$, corrected).

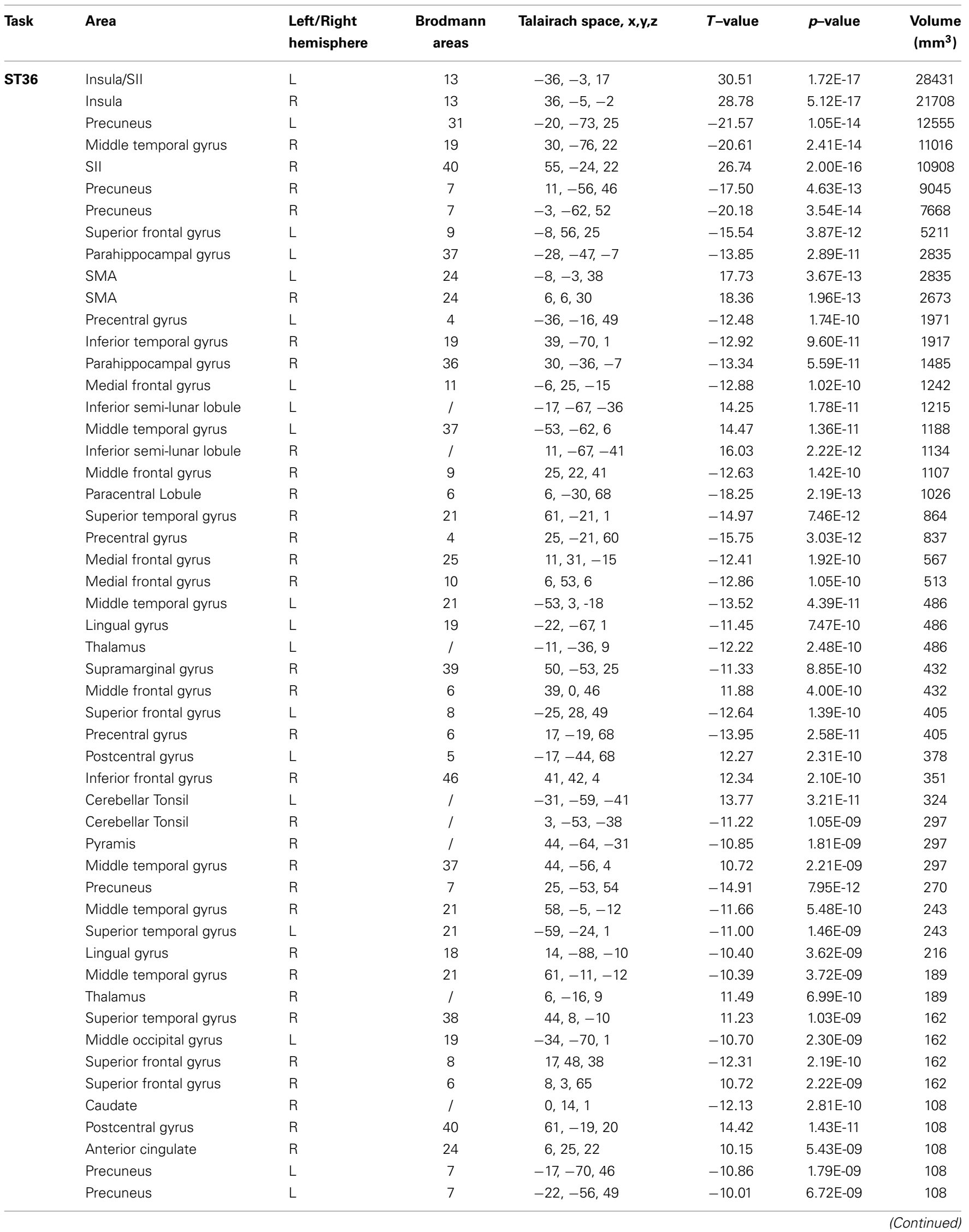


Table 1 | Continued

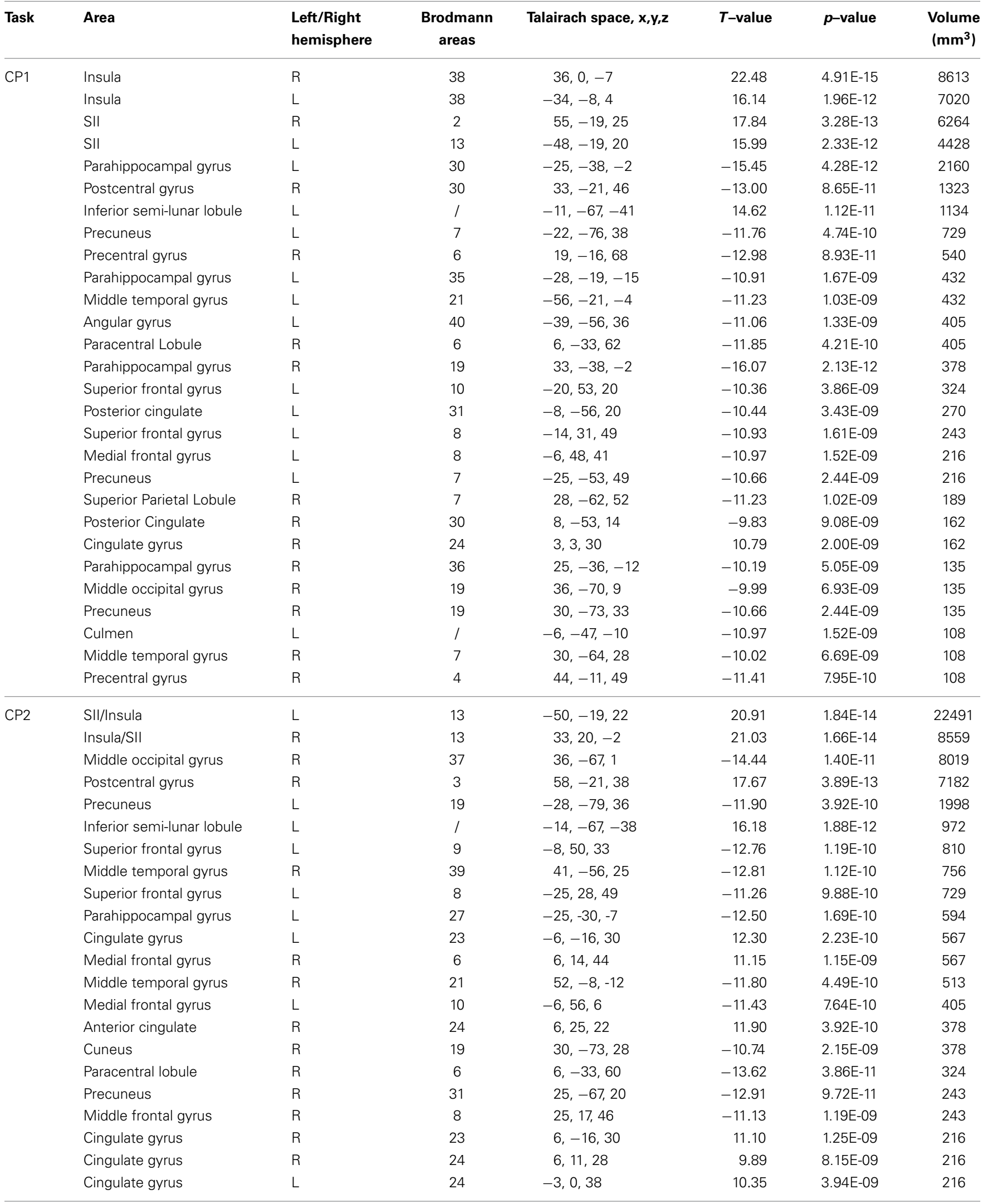


Table 1 | Continued

\begin{tabular}{|c|c|c|c|c|c|c|c|}
\hline Task & Area & $\begin{array}{l}\text { Left/Right } \\
\text { hemisphere }\end{array}$ & $\begin{array}{l}\text { Brodmann } \\
\text { areas }\end{array}$ & Talairach space, $x, y, z$ & $T$-value & $p$-value & $\begin{array}{c}\text { Volume } \\
\left(\mathrm{mm}^{3}\right)\end{array}$ \\
\hline & Parahippocampal gyrus & $\mathrm{R}$ & 34 & $28,-11,-15$ & -11.96 & $3.60 \mathrm{E}-10$ & 189 \\
\hline & Postcentral gyrus & $\mathrm{R}$ & 3 & $22,-24,49$ & -10.99 & 1.47E-09 & 162 \\
\hline & Declive & $\mathrm{R}$ & l & $17,-67,-15$ & 10.20 & 4.97E-09 & 135 \\
\hline & Caudate & $\mathrm{R}$ & l & $8,6,12$ & 10.29 & 4.32E-09 & 108 \\
\hline
\end{tabular}

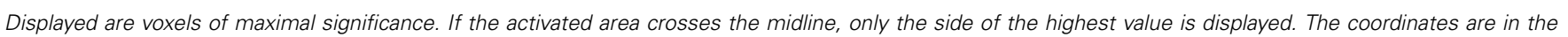
Talairach space. T and $p$ value is on the voxel-level.

observed in the follow-up resting-state scan for the comparisons of ST36 with the two control points, but in different regions of right precuneus. These results suggest differential processing of acupuncture point stimulation compared to stimulation of nonacupuncture control points, including a potential mechanism of pain modulation due to a complex somatosensory stimulation.

To answer our focused research question we applied a rigorous study design. The subjects were blinded regarding the character of the different point locations and the researchers were blinded during the pre-processing of the data and during the first steps of data interpretation. To prevent systematic errors, different randomization procedures were used. The order of point locations was randomized for both experiments, and the interstimulus intervals were randomized during the fMRI experiment. The washout period of acupuncture stimulation is still unknown. By randomizing the order of point locations all three interventions should be comparably affected by possible carry-over effects. A broad range of somatosensory effects were assessed using EEG, BOLD, and resting-state fcMRI data analysis. Thus, we evaluated event-related changes as well as longer lasting brain activity changes (connectivity and EEG rhythm). Event-related designs can robustly image brain response to discrete, short duration acupuncture stimuli (Napadow et al., 2012) which correspond well with the clinical application of acupuncture stimulation.

Manual acupuncture was chosen because it is more relevant for the clinical setting. But this might also be a cause of systematic error, because acupuncturists obviously could not be blinded in our study. Therefore, we evaluated the needle sensations as reported by the subjects. In part, needle sensations were different between the stimulated points, but we found that sensation was not correlated with brain activity changes. All subjects received the stimulation on all three point locations, therefore the groups we compared were based only on different point locations not on different subjects. We used intra-individual comparisons because the variance of physiological parameters between subjects is typically more pronounced than intra-subject differences caused by an intervention like a somatosensory stimulation on three different points. Because of intra-individual comparisons our data was not independent, though this was taken into account during our statistical analysis. Moreover, age and gender were included into the statistical models as covariates, since these factors might influence the outcome when evaluating the effects of acupuncture. In general, with the subtractive design used in our study, possible interferences between acupuncture effects and the somatotopic organization of evoked brain responses cannot be fully disentangled. This question could be addressed with a $2 \times 2$ factorial design with two pairs of acupuncture point and control point in different dermatomes, though involving additional experimental effort.

The results of the needle sensation for the two experiments were not comparable. In the EEG experiment the MASS index for ST36 stimulation was similar to CP1 and different from CP2. However, in the fMRI experiment the MASS index for ST36 stimulation was significantly different from the two control points. Several conditions might explain the differences. The position of participants in the two experiments were different. In the EEG experiment, participants were in a sitting position while in a supine position in the fMRI experiment, where the subjects might feel more relaxed than in a sitting position. Moreover, muscle tension on the leg where the needling stimulation was applied can be different in these two positions, and thus influence the sensation processing. In addition, a finer needle (different material and size) had to be used in the fMRI experiment because of the magnetic field of the scanner. Due to the repeated intermittent stimulation in the event-related design of the fMRI experiment, the stimulation protocol might have been more intense than in the EEG experiment.

Many studies show that various forms of somatosensory stimulation (from light touch to painful stimuli) cause transient desynchronization (suppression) of the somatosensory (mu) background rhythm (Neuper et al., 2006; Ploner et al., 2006b; Stancak, 2006). In our study, we observed increased mu rhythm following needle stimulation. Until now, to our best knowledge, such an after-effect of a peripheral somatosensory stimulation has not been described in the literature. A recent EEG study indicated that acupuncture stimulation on acupuncture point LI4 seemed to lead to specific changes in alpha EEG-frequency (Streitberger et al., 2008). However, the authors compared manual penetrating acupuncture with nonpenetrating needle stimulation on the same point, i.e., they compared different kinds of stimulation rather than different points. Similar effects of long lasting increased background rhythm have been described for non-invasive brain stimulation protocols such as TDCS/TACS (transcranial direct/alternating 
Table 2 | BOLD changes: a comparison of the three points showing all significant contrasts $(P<0.05$, corrected).

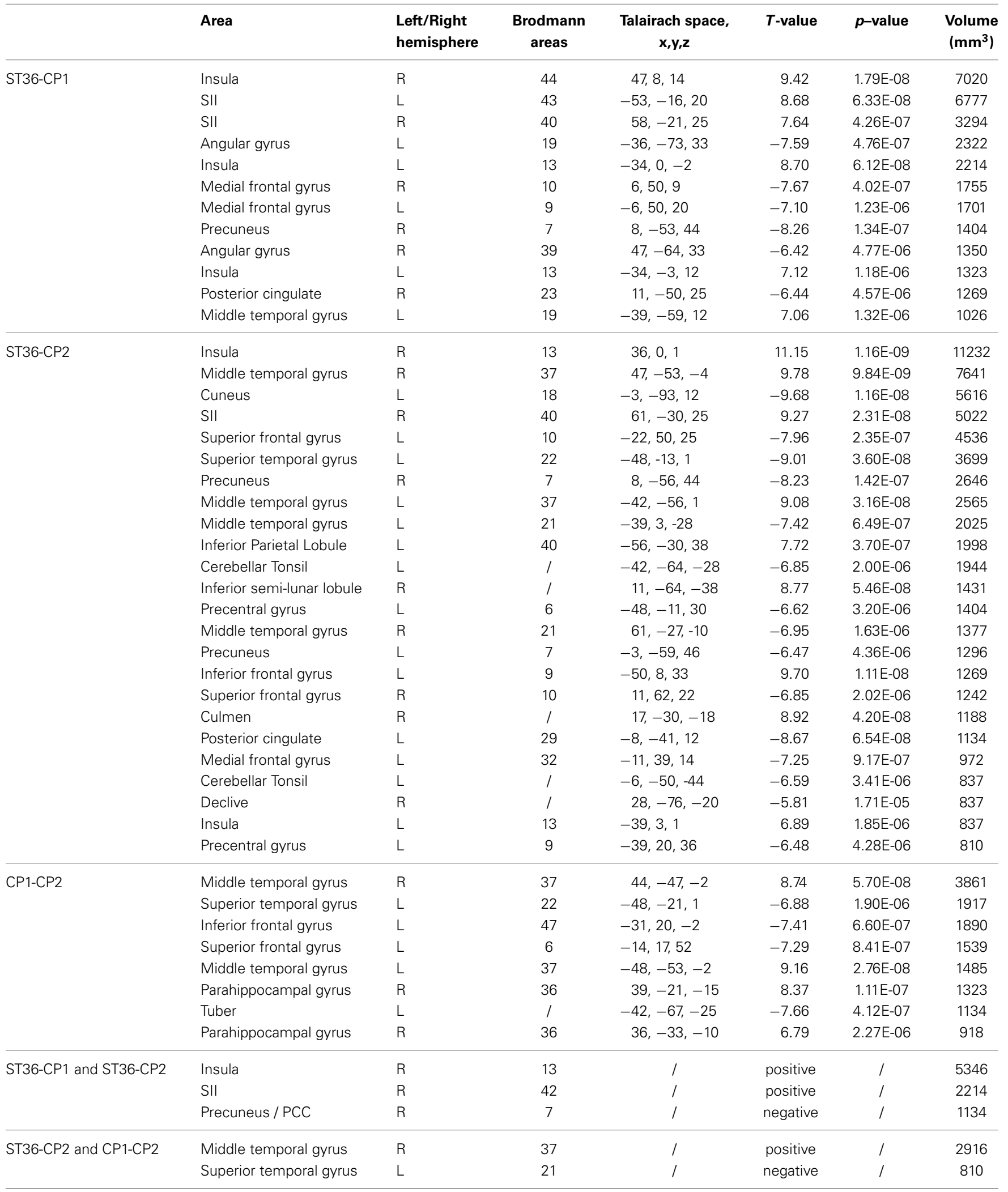

Displayed are voxels of maximal significance. If the activated area crosses the midline, only the side of the highest value is displayed. The coordinates are in the Talairach space. T and $p$ value is on the voxel-level. The results of the conjunction analysis are also listed. "Positive" means ST36 presented higher activity than two control points, and vice versa. 
Table 3 | Brain regions which were detected on the group level in the somatosensory network analysis from all resting-state sessions ( $P<0.05$, corrected).

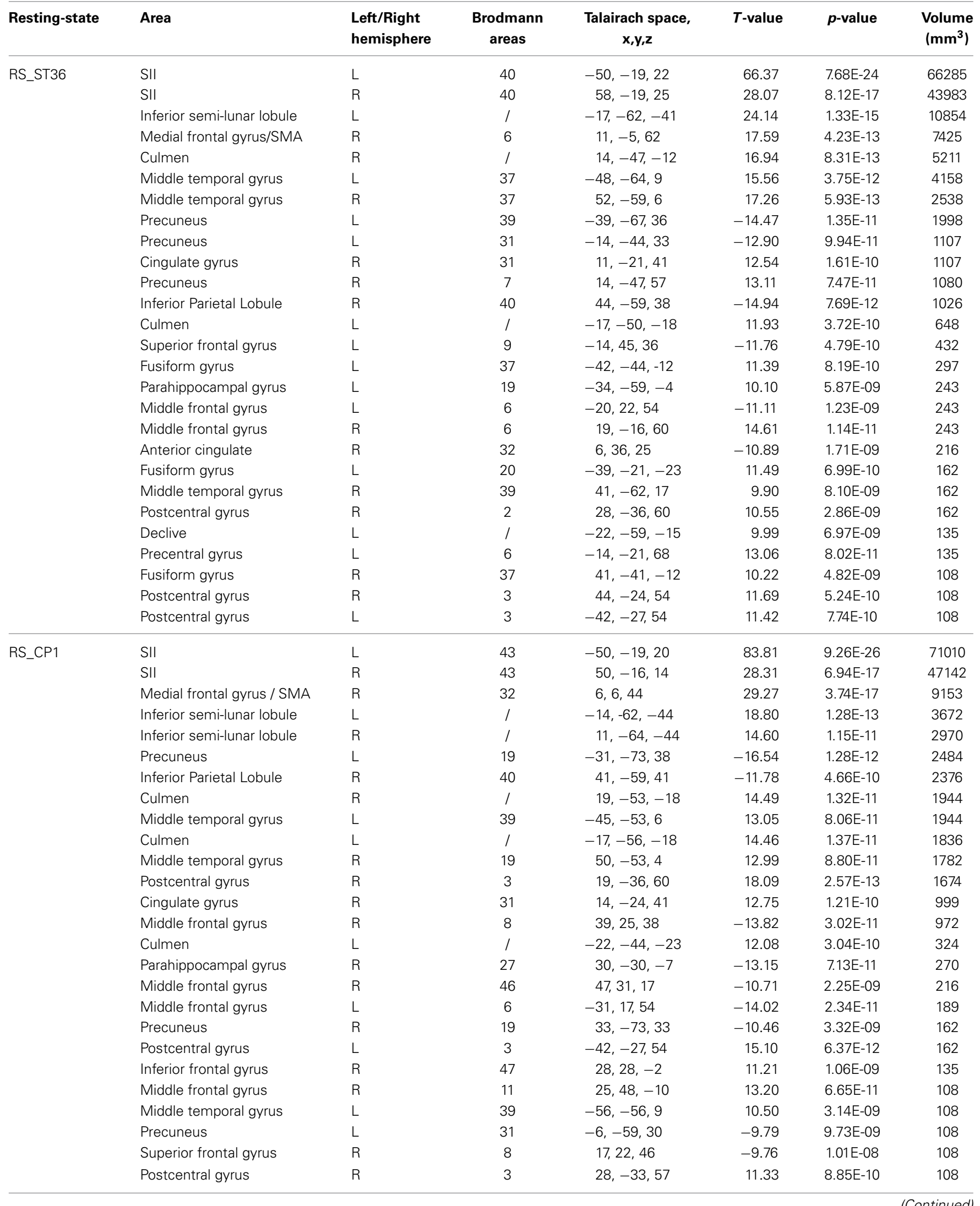


Table 3 | Continued

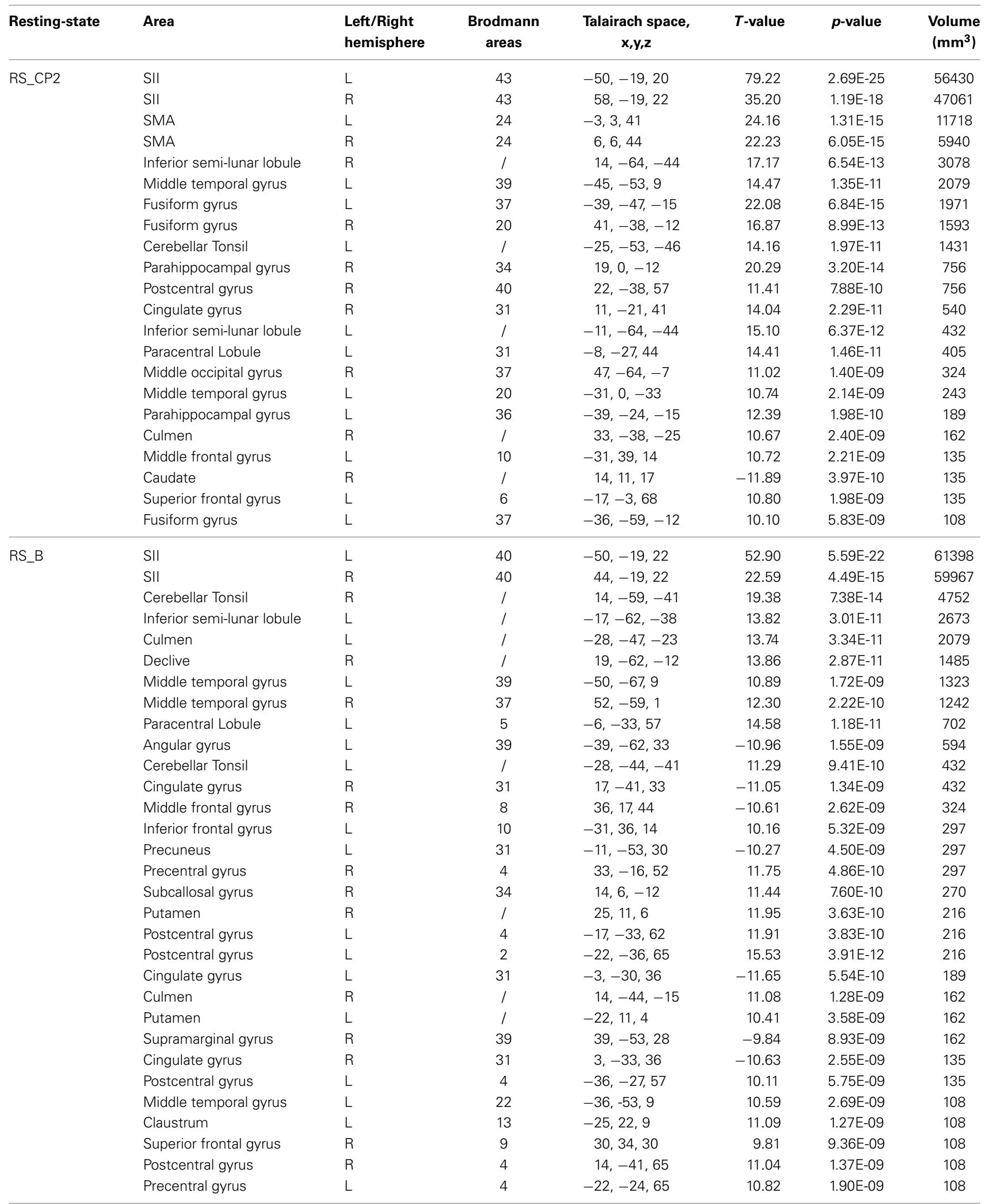

Displayed are voxels of maximal significance. If the activated area crosses the midline, only the side of the highest value is displayed. The coordinates are in the Talairach space. $T$ and $p$ value is on the voxel-level. 


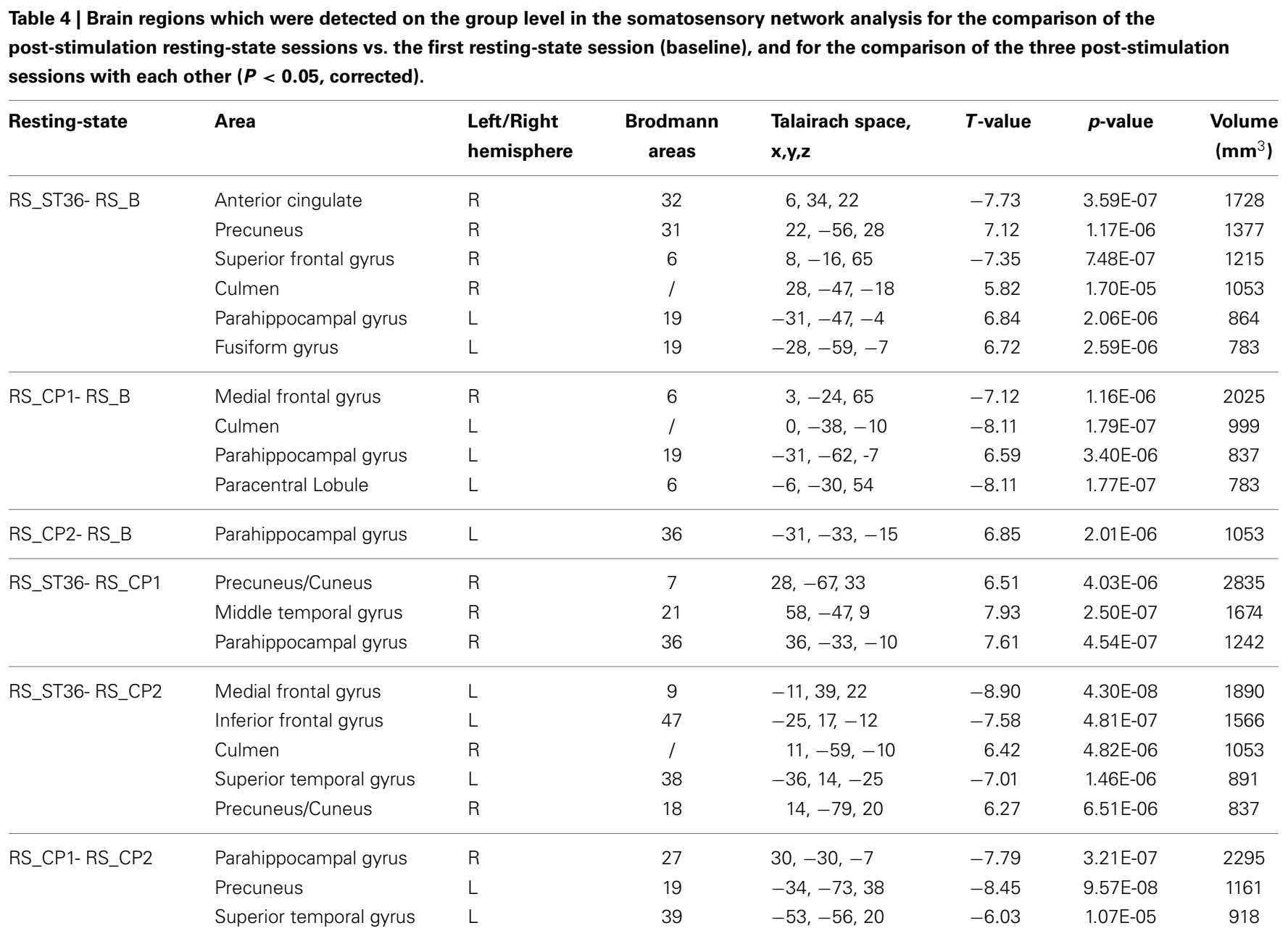

Displayed are voxels of maximal significance. If the activated area crosses the midline, only the side of the highest value is displayed. The coordinates are in the Talairach space. T and $p$ value is on the voxel-level.

current stimulation) or TMS (transcranial magnetic stimulation) (Wagner et al., 2007).

The BOLD activation pattern and connectivity changes we found in our fMRI analysis correspond well with previous findings showing that acupuncture stimulation modulates activity and connectivity of somatosensory as well as pain-related areas (especially insula cortex and S2). Recent neuroimaging studies that compared the stimulation of acupuncture points to control points revealed strengthened BOLD activation in somatosensory areas, the cingulum, the basal ganglia, the brainstem, the cerebellum, as well as the insula cortex. Besides these increases in BOLD activation, these studies also found pronounced acupuncture related deactivation of BOLD signaling in the amygdala, the hippocampus, and brain areas well described as hubs of the brain's default mode network (Dhond et al., 2007; Huang et al., 2012). These observations are in good agreement with the present findings since we also found acupuncture related deactivation in default mode network associated areas and higher BOLD activation in S2 and insula, which are well described as dominant hubs of the central nervous pain network (also known as pain matrix Apkarian et al., 2005; May, 2007). Furthermore, for ST36 as compared to the control points, we found a significantly deactivated right precuneus during stimulation. Although no voxels survived the conjunction analysis for the connectivity comparison of acupuncture point vs. control points, both comparisons (RS_ST36-RS_CP1 and RS_ST36-RS_CP2) showed increased connectivity between right precuneus and S2 in the follow-up resting-state scan. Whereas S2 and insula are assumed to contribute to the experience of pain (Craig, 2009), the precuneus seems to be involved in the assessment and integration of pain (Goffaux et al., 2014). Acupuncture related strengthened functional connectivity between S2 and precuneus might represent a possible mechanism that explains the pain relieving effectiveness of acupuncture, especially in chronic pain (Berman et al., 2010; Vickers et al., 2012). Additionally, our finding of an increased mu rhythm following stimulation of ST36 may represent another potential mechanisms of pain modulation, since an increased mu rhythm was previously shown to be associated with a pronounced cortical inhibition (Klimesch et al., 2007; Jensen and Mazaheri, 2010) and a reduced cortical excitability to painful stimulation (Ploner et al., 2006a). Further studies combining acupuncture with multimodal brain imaging are necessary 


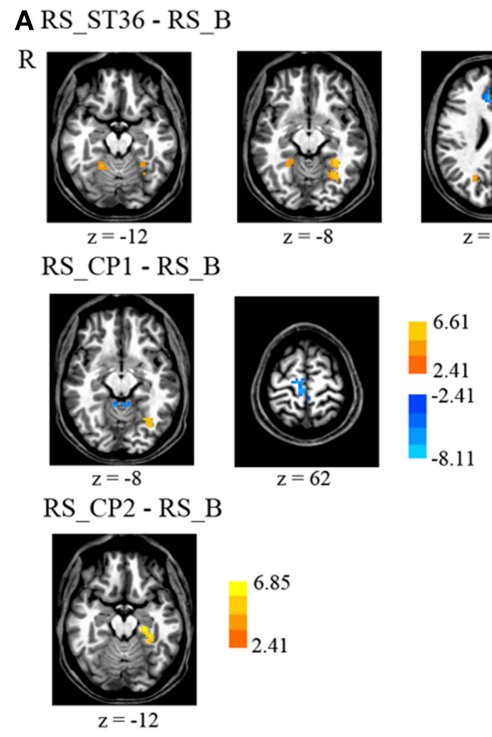

FIGURE 4 | Comparison of the seed-based somatosensory resting-state connectivity between all resting-state sessions $(P<\mathbf{0 . 0 5}$, corrected). (A) The comparison of post-stimulation resting-state scans with the baseline scan. (B) The comparison of the post-stimulation resting-state scans with
B RS ST36-RS CP1
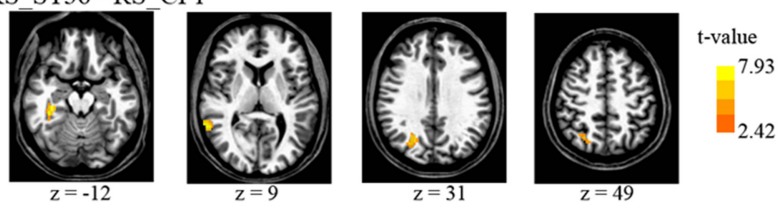

RS ST36 - RS CP2
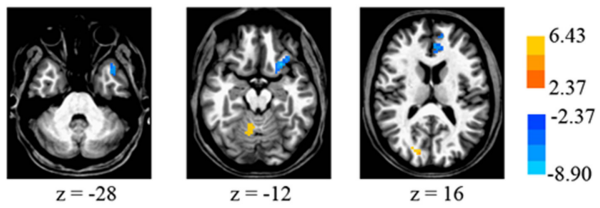

RS_CP1 - RS_CP2
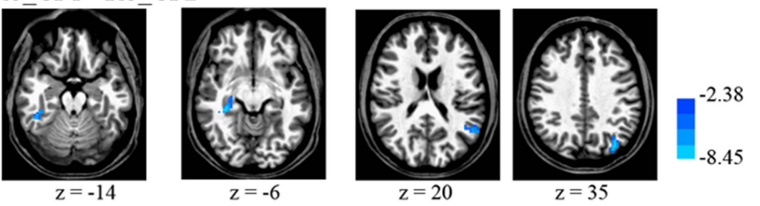

each other. There is no cluster surviving the conjunction analyses across the first and second row (acupuncture vs. control points) as well as across the second and the third row (dermatome L5 vs. L2). R means right hemisphere. Talairach z coordinates are displayed. positive values: red, negative: blue. to test these hypotheses in patients suffering from chronic pain.

Furthermore, we found a pronounced activation at right middle temporal gyrus and deactivation at left superior temporal gyrus during needle stimulation, which was also found by other studies that investigate acupuncture (Zhang et al., 2012; Kim et al., 2013). However, we found these effects only for the comparison between the different dermatomes (L2 and L5) when stimulating dermatome L5 (ST36 or CP1). These effects might hint toward a different sensitivity of the two different regions used for the needle stimulation, but still remain elusive.

In conclusion, our findings suggest that stimulation at acupuncture points may modulate somatosensory and saliency processing regions more readily than stimulation at nonacupuncture point locations. In addition, our results hint toward potential mechanisms of pain modulation due to acupuncture stimulation. Furthermore, our results might have an impact on experiments using conventional somatosensory stimulation protocols. For example, electrical stimulation applied to the median nerve or the finger might produce different imaging results when the stimulation electrodes are located near an acupuncture point. Further experiments using electrical acupuncture and EEG will assess if direct stimulation of acupuncture points also affects established EEG markers, such as evoked potentials or evoked and induced rhythmic activity.

\section{FUNDING}

This study had no additional funding. Wenjing Huang received a scholarship from the Carstens Foundation. Vitaly Napadow was supported by NCCAM, National Institutes of Health [R01-AT004714, R01-AT005280, P01-AT006663, R21-DK097499, R01-AT007550].

\section{AUTHOR CONTRIBUTIONS}

Conceived and designed the experiments: TN, WH, DP, CMW, AV, VN, FL. Performed the trial: TN, WH, DP. Analyzed the data: TN, XL, SR. Discussed the data: TN, WH, DP, XL, CMW, AV, VN, BP, SR, FL. Wrote the first draft of the paper: TN, DP, WH. Revised the paper and approved the final version: TN, WH, DP, CMW, AV, VN, SR, BP, FL.

\section{ACKNOWLEDGMENTS}

We thank Sylvia Stasch and Annett Wiedemann for their support. We also thank the reviewers' generous suggestions.

\section{SUPPLEMENTARY MATERIAL}

The Supplementary Material for this article can be found online at: http://www.frontiersin.org/journal/10.3389/fnhum. 2015.00074/abstract

\section{REFERENCES}

Apkarian, A. V., Bushnell, M. C., Treede, R. D., and Zubieta, J. K. (2005). Human brain mechanisms of pain perception and regulation in health and disease. Eur. J. Pain 9, 463-484. doi: 10.1016/j.ejpain.2004.11.001

Bäcker, M., Hammes, M., Sander, D., Funke, D., Deppe, M., Tolle, T. R., et al. (2004). Changes of cerebrovascular response to visual stimulation in migraineurs after repetitive sessions of somatosensory stimulation (acupuncture): a pilot study. Headache 44, 95-101. doi: 10.1111/j.15264610.2004.04017.x

Bandettini, P. A., Wong, E. C., Hinks, R. S., Tikofsky, R. S., and Hyde, J. S. (1992). Time course EPI of human brain function during task activation. Magn. Reson. Med. 25, 390-397.

Behzadi, Y., Restom, K., Liau, J., and Liu, T. T. (2007). A component based noise correction method (CompCor) for BOLD and perfusion based fMRI. Neuroimage 37, 90-101. doi: 10.1016/j.neuroimage.2007.04.042

Berman, B. M., Langevin, H. M., Witt, C. M., and Dubner, R. (2010). Acupuncture for chronic low back pain. New Engl. J. Med. 363, 454-461. doi: 10.1056/NEJMct0806114 
Brinkhaus, B., Becker-Witt, C., Jena, S., Linde, K., Streng, A., Wagenpfeil, S., et al. (2003). Acupuncture Randomized Trials (ART) in patients with chronic low back pain and osteoarthritis of the knee - design and protocols. Forsch. Komplementarmed. Klass. Naturheilkd. 10, 185-191. doi: 10.1159/0000 73474

Cox, R. W. (1996). AFNI: software for analysis and visualization of functional magnetic resonance neuroimages. Comput. Biomed. Res. 29, $162-173$.

Craig, A. D. (2009). How do you feel-now? The anterior insula and human awareness. Nat. Rev. Neurosci. 10, 59-70. doi: 10.1038/nrn2555

Dhond, R. P., Kettner, N., and Napadow, V. (2007). Neuroimaging acupuncture effects in the human brain. J. Altern. Complement. Med. 13, 603-616. doi: 10.1089/acm.2007.7040

Dhond, R. P., Yeh, C., Park, K., Kettner, N., and Napadow, V. (2008). Acupuncture modulates resting state connectivity in default and sensorimotor brain networks. Pain 136, 407-418. doi: 10.1016/j.pain.2008.01.011

Diener, H. C., Kronfeld, K., Boewing, G., Lungenhausen, M., Maier, C., Molsberger, A., et al. (2006). Efficacy of acupuncture for the prophylaxis of migraine: a multicentre randomised controlled clinical trial. Lancet Neurol. 5, 310-316. doi: 10.1016/S1474-4422(06)70382-9

Drake, R., Vogl, A. W., and Mitchell, A. W. (2009). Gray's Anatomy For Students. London: Elsevier Health Sciences.

Fox, M. D., Snyder, A. Z., Vincent, J. L., Corbetta, M., Van Essen, D. C., and Raichle, M. E. (2005). The human brain is intrinsically organized into dynamic, anticorrelated functional networks. Proc. Natl. Acad. Sci. U.S.A. 102, 9673-9678. doi: 10.1073/pnas.0504136102

Frahm, J., Bruhn, H., Merboldt, K. D., and Hanicke, W. (1992). Dynamic MR imaging of human brain oxygenation during rest and photic stimulation. J. Magn. Reson. Imaging 2, 501-505.

Gastaut, H. (1952). Électrocorticographique de la Réactivité des Rhythmes Rolandiques. Rev. Neurol. Paris 87, 176-182.

Goffaux, P., Girard-Tremblay, L., Marchand, S., Daigle, K., and Whittingstall, K. (2014). Individual differences in pain sensitivity vary as a function of precuneus reactivity. Brain Topogr. 27, 366-374. doi: 10.1007/s10548-0130291-0

Greicius, M. D., Flores, B. H., Menon, V., Glover, G. H., Solvason, H. B., Kenna, H., et al. (2007). Resting-state functional connectivity in major depression: abnormally increased contributions from subgenual cingulate cortex and thalamus. Biol. Psychiatry 62, 429-437. doi: 10.1016/j.biopsych.2006.09.020

Hori, E., Takamoto, K., Urakawa, S., Ono, T., and Nishijo, H. (2010). Effects of acupuncture on the brain hemodynamics. Auton. Neurosci. 157, 74-80. doi: 10.1016/j.autneu.2010.06.007

Huang, W., Pach, D., Napadow, V., Park, K., Long, X., Neumann, J., et al. (2012). Characterizing acupuncture stimuli using brain imaging with FMRI-a systematic review and meta-analysis of the literature. PLoS ONE 7:e32960. doi: 10.1371/journal.pone.0032960

Hui, K. K., Liu, J., Makris, N., Gollub, R. L., Chen, A. J., Moore, C. I., et al. (2000). Acupuncture modulates the limbic system and subcortical gray structures of the human brain: evidence from fMRI studies in normal subjects. Hum. Brain Mapp. 9, 13-25. doi: 10.1002/(SICI)1097-0193(2000)9:1\%3C13::AIDHBM2\%3E3.0.CO;2-F

Jensen, O., and Mazaheri, A. (2010). Shaping functional architecture by oscillatory alpha activity: gating by inhibition. Front. Hum. Neurosci. 4:186. doi: 10.3389/fnhum.2010.00186

Kim, N. H., Cho, S. Y., Jahng, G. H., Ryu, C. W., Park, S. U., Ko, C. N., et al. (2013) Differential localization of pain-related and pain-unrelated neural responses for acupuncture at BL60 using BOLD fMRI. Evid. Based Complement. Alternat. Med. 2013:804696. doi: 10.1155/2013/804696

Kleinhenz, J., Streitberger, K., Windeler, J., Gussbacher, A., Mavridis, G., and Martin, E. (1999). Randomised clinical trial comparing the effects of acupuncture and a newly designed placebo needle in rotator cuff tendinitis. Pain 83, 235-241.

Klimesch, W., Sauseng, P., and Hanslmayr, S. (2007). EEG alpha oscillations: the inhibition-timing hypothesis. Brain Res. Rev. 53, 63-88. doi: 10.1016/j.brainresrev.2006.06.003

Kong, J., Gollub, R., Huang, T., Polich, G., Napadow, V., Hui, K., et al. (2007). Acupuncture de qi, from qualitative history to quantitative measurement. J. Altern. Complement. Med. 13, 1059-1070. doi: 10.1089/acm.2007.0524
Kuhlman, W. N. (1978). Functional topography of the human mu rhythm. Electroencephalogr. Clin. Neurophysiol. 44, 83-93.

Kurth, R., Villringer, K., Curio, G., Wolf, K. J., Krause, T., Repenthin, J., et al. (2000). fMRI shows multiple somatotopic digit representations in human primary somatosensory cortex. Neuroreport 11, 1487-1491. doi: 10.1097/00001756200005150-00025

Kwong, K. K., Belliveau, J. W., Chesler, D. A., Goldberg, I. E., Weisskoff, R. M., Poncelet, B. P., et al. (1992). Dynamic magnetic resonance imaging of human brain activity during primary sensory stimulation. Proc. Natl. Acad. Sci. U.S.A. 89, 5675-5679.

Linde, K., Streng, A., Hoppe, A., Brinkhaus, B., Becker-Witt, C., Hammes, M., et al. (2006). Treatment in a randomized multicenter trial of acupuncture for migraine (ART Migraine). Forsch. Komplementärmed 13, 101-108. doi: $10.1159 / 000091999$

Ma, T. T., Yu, S. Y., Li, Y., Liang, F. R., Tian, X. P., Zheng, H., et al. (2012). Randomised clinical trial: an assessment of acupuncture on specific meridian or specific acupoint vs. sham acupuncture for treating functional dyspepsia. Aliment. Pharmacol. Ther. 35, 552-561. doi: 10.1111/j.1365-2036.2011. 04979.x

May, A. (2007). Neuroimaging: visualising the brain in pain. Neurol. Sci. 28(Suppl. 2), S101-S107. doi: 10.1007/s10072-007-0760-x

Melchart, D., Streng, A., Hoppe, A., Brinkhaus, B., Witt, C., Wagenpfeil, S., et al. (2005). Acupuncture in patients with tension-type headache: randomised controlled trial. BMJ 331, 376-382. doi: 10.1136/bmj.38512.40 $5440.8 \mathrm{~F}$

Napadow, V., Dhond, R., Park, K., Kim, J., Makris, N., Kwong, K. K., et al. (2009). Time-variant fMRI activity in the brainstem and higher structures in response to acupuncture. Neuroimage 47, 289-301. doi: 10.1016/j.neuroimage.2009.03.060

Napadow, V., Lee, J., Kim, J., Cina, S., Maeda, Y., Barbieri, R., et al. (2012). Brain correlates of phasic autonomic response to acupuncture stimulation: an event-related fMRI study. Hum. Brain Mapp. 34, 2592-2606. doi: 10.1002/hbm. 22091

Neuper, C., Wortz, M., and Pfurtscheller, G. (2006). ERD/ERS patterns reflecting sensorimotor activation and deactivation. Prog. Brain Res. 159, 211-222. doi: 10.1016/S0079-6123(06)59014-4

Ogawa, S., Tank, D. W., Menon, R., Ellermann, J. M., Kim, S. G., Merkle, H., et al. (1992). Intrinsic signal changes accompanying sensory stimulation: functional brain mapping with magnetic resonance imaging. Proc. Natl. Acad. Sci. U.S.A. $89,5951-5955$.

Oldfield, R. C. (1971). The assessment and analysis of handedness: the Edinburgh inventory. Neuropsychologia 9, 97-113.

Ploner, M., Gross, J., Timmermann, L., Pollok, B., and Schnitzler, A. (2006a). Oscillatory activity reflects the excitability of the human somatosensory system. Neuroimage 32, 1231-1236. doi: 10.1016/j.neuroimage.2006. 06.004

Ploner, M., Gross, J., Timmermann, L., Pollok, B., and Schnitzler, A. (2006b). Pain suppresses spontaneous brain rhythms. Cereb. Cortex 16, 537-540. doi: 10.1093/cercor/bhj001

Ritter, P., Moosmann, M., and Villringer, A. (2009). Rolandic alpha and beta EEG rhythms' strengths are inversely related to fMRI-BOLD signal in primary somatosensory and motor cortex. Hum. Brain Mapp. 30, 1168-1187. doi: 10.1002/hbm.20585

Ruben, J., Schwiemann, J., Deuchert, M., Meyer, R., Krause, T., Curio, G., et al. (2001). Somatotopic organization of human secondary somatosensory cortex. Cereb. Cortex 11, 463-473. doi: 10.1093/cercor/11.5.463

Salmelin, R., and Hari, R. (1994). Spatiotemporal characteristics of sensorimotor neuromagnetic rhythms related to thumb movement. Neuroscience 60, 537-550. doi: 10.1016/0306-4522(94)90263-1

Sanchez-Araujo, M. (1998). Does the choice of placebo determine the results of clinical studies on acupuncture? Forsch. Komplementarmed. 5, 8-11.

Stancak, A. (2006). Cortical oscillatory changes occurring during somatosensory and thermal stimulation. Prog. Brain Res. 159, 237-252. doi: 10.1016/S00796123(06)59016-8

Streitberger, K., Steppan, J., Maier, C., Hill, H., Backs, J., and Plaschke, K. (2008). Effects of verum acupuncture compared to placebo acupuncture on quantitative EEG and heart rate variability in healthy volunteers. J. Altern. Complement. Med. 14, 505-513. doi: 10.1089/acm.2007.0552 
Vickers, A. J., Cronin, A. M., Maschino, A. C., Lewith, G., Macpherson, H., Foster, N. E., et al. (2012). Acupuncture for chronic pain: individual patient data metaanalysis. Arch. Int. Med. 1-10. doi: 10.1001/archinternmed.2012.3654

Wagner, T., Valero-Cabre, A., and Pascual-Leone, A. (2007). Noninvasive human brain stimulation. Annu. Rev. Biomed. Eng. 9, 527-565. doi: 10.1146/annurev.bioeng.9.061206.133100

Who Regional Office for the Western Pacific. (2008). WHO Standard Acupuncture Point Locations in the Western Pacific Region. Manila: World Health Organization.

Yan, Z. (2006). Anatomy of Acupuncture Points and Clinical Applications. Shanghai: Publishing House of Shanghai University of TCM.

Yang, X., Li, Y., Tian, X., and Liang, F. (2009). Locations and evaluations of nonacupuncture points in acupuncture studys from China and Abroad. J. Tradit. Chin. Med. 50, 748-750.

Zhang, Y., Glielmi, C. B., Jiang, Y., Wang, J., Wang, X., Fang, J., et al. (2012). Simultaneous CBF and BOLD mapping of high frequency acupuncture induced brain activity. Neurosci. Lett. 530, 12-17. doi: 10.1016/j.neulet.2012.09.050
Conflict of Interest Statement: The authors declare that the research was conducted in the absence of any commercial or financial relationships that could be construed as a potential conflict of interest.

Received: 25 July 2014; accepted: 29 January 2015; published online: 13 February 2015. Citation: Nierhaus T, Pach D, Huang W, Long X, Napadow V, Roll S, Liang F, Pleger $B$, Villringer $A$ and Witt CM (2015) Differential cerebral response to somatosensory stimulation of an acupuncture point vs. two non-acupuncture points measured with EEG and fMRI. Front. Hum. Neurosci. 9:74. doi: 10.3389/fnhum.2015.00074

This article was submitted to the journal Frontiers in Human Neuroscience. Copyright (C) 2015 Nierhaus, Pach, Huang, Long, Napadow, Roll, Liang, Pleger, Villringer and Witt. This is an open-access article distributed under the terms of the Creative Commons Attribution License (CC BY). The use, distribution or reproduction in other forums is permitted, provided the original author(s) or licensor are credited and that the original publication in this journal is cited, in accordance with accepted academic practice. No use, distribution or reproduction is permitted which does not comply with these terms. 